Proceedings

\title{
Detection of Transverse Defects in Rails Using Noncontact Laser Ultrasound ${ }^{+}$
}

\author{
Hajar Benzeroual 1,*, Abdellatif Khamlichi ${ }^{2}$ and Alia Zakriti ${ }^{2}$ \\ 1 Systems of Communication and Detection Laboratory, Department of Physics, Faculty of Sciences, \\ 93000 Tetouan, Morocco \\ 2 Computer Department of Industrial and Civil Engineering Sciences, National School of Applied Sciences, \\ 93000 Tetouan, Morocco; khamlichi7@yahoo.es (A.K.); alia_zakriti@yahoo.fr (A.Z.) \\ * Correspondence: benzeroual.hajar@gmail.com \\ + Presented at the 6th International Electronic Conference on Sensors and Applications, 15-30 November \\ 2019; Available online: https://ecsa-6.sciforum.net/.
}

Published: 14 November 2019

\begin{abstract}
Rail inspections are required and used to ensure safety and preserve the availability of railway infrastructure. According to the statistics published by railroad administrations worldwide, the transverse fissure appearing in railhead is the principal cause of rail accidents. These particular defects are initiated inside the railhead. Detection of these cracks has always been challenging because a defect signature remains mostly small until the defect size reaches a significant value. The present work deals with the theoretical analysis of an integrated contact-less system for rail diagnosis, which is based on ultrasounds. The generation of these waves was performed through non-ablative laser sources. Rotational laser vibrometry was used to achieve the reception of the echoes. Detection of flaws in the rail was monitored by considering special ultrasound wave signal based indicators. Finite element modeling of the rail system was performed, and transverse defect detection of the rail was analyzed.
\end{abstract}

Keywords: rail; transverse fissure; contact-less system; ultrasound; laser; rotational laser vibrometry

\section{Introduction}

Historically, rail inspections were done visually. During the past century, various detection methods were introduced to determine the state of rail health, even if defects had not yet reached the rail surface. These methods included eddy-currents, infrared thermography, and ultrasonics [1-3]. The ultrasonic technique was recognized to be suitable for operating the detection of transverse like flaws. The early detection of these defects constitutes a challenge for improving the security of railways and preventing catastrophic failures.

Ultrasonic based inspection techniques for railways utilize the phenomenon of elastic wave propagation. The excitation method can be performed using a contactless pulse-echo configuration, based on laser-generated ultrasound. The reflection of elastic waves may occur at a defect present in the rail system, and the generated echo can then be sensed. A contact-less ultrasound sensor consisting of a rotational laser vibrometer that performs reception of reflected echoes from defects is considered. The simulation of ultrasonic wave propagation was done on ABAQUS software. The wave is assumed to be generated from a laser-irradiated spot, located on the railhead. The reception of rail response is performed by using a laser vibrometer sensor.

The purpose of this work is to identify the optimal position of the sensor to capture relevant information and improve the detectability of flaws as early as possible. 


\section{Materials and Methods}

\subsection{Material Properties}

The rail structure considered in this work was made from a linear homogeneous and isotropic elastic steel material. The total length of the rail was $\mathrm{L}=600 \mathrm{~mm}$. The position of a localized transverse defect was halfway between the transmitter and the receiver, as shown in Figure 1. The material properties are given in Table 1.

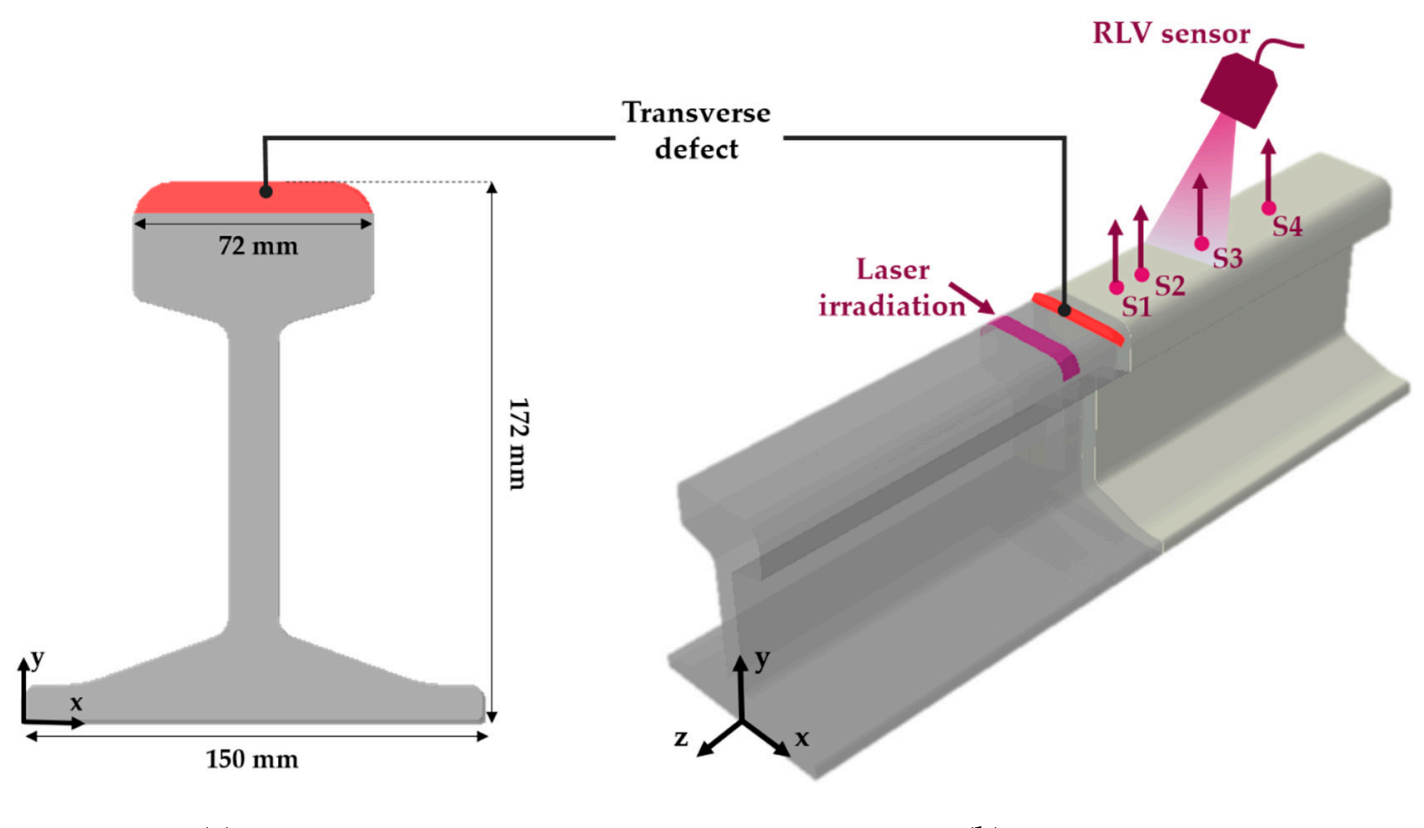

(a)

(b)

Figure 1. (a) The geometry of the rail 60E1; (b) The localization of rotational laser vibrometry (RLV) reception sensors, transverse defects, and the spot excitation zones for rail inspection.

Table 1. Material properties of the rail structure.

\begin{tabular}{cc}
\hline Material Properties & Value \\
\hline Young's Modulus (GPa) & 210 \\
Density $\left(\mathrm{kg} / \mathrm{m}^{3}\right)$ & 7800 \\
Poisson's ratio & 0.3 \\
Conductivity $(\mathrm{W} /(\mathrm{m} . \mathrm{K}))$ & 50.2 \\
Specific Heat $(\mathrm{J} / \mathrm{kg} \mathrm{K})$ & 490 \\
\hline
\end{tabular}

\subsection{Defect Geometry}

Transverse defects can range from surface defects to internal cracks [4]. The geometry of the studied defect was rectangular and $5 \mathrm{~mm}$ in depth. Table 2 shows the parameters of such a defect.

Table 2. Parameters of transverse defect located in the rail.

\begin{tabular}{cccc}
\hline Defect Volume in $\left(\mathbf{m m}^{3}\right)$ & Defect Length in $\mathbf{x}$ & Defect Width in $\mathrm{z}$ & Defect Depth in $\mathbf{y}$ \\
\hline 700 & $72 \mathrm{~mm}$ & $2 \mathrm{~mm}$ & $5 \mathrm{~mm}$ \\
\hline
\end{tabular}

The detection of this type of flaw has been monitored through a special ultrasound device that generates ultrasonic signals using short, focused laser irradiation on the top head of the rail, and senses the back-reflected echo. 


\subsection{Rail Inspection Method}

The method of excitation uses a laser ultrasound transducer that includes a single laser spot irradiation to excite the railhead. In this case, the spot size was chosen to be $15 \mathrm{~mm}$ in width and 72 $\mathrm{mm}$ in length.

For the reception of wave signals, a termed rotational laser vibrometry (RLV) sensor was used to measure the deformation caused by propagating ultrasonic waves. RLV is a contact-less device that allowed us to very accurately measure the angular velocity and the angular displacement generated by elastic deformation on the rail structure. In this work, the RLV was calibrated to measure the normal velocity with the y-direction at several positions of the rail, as can be shown in Figure 1.

\section{Results and Discussion}

A finite element model of the proposed diagnostic device was developed using ABAQUS software. The model was simulated for a rail with a defect and an intact rail.

The use of sensors here was designed to determine the normal velocity at each chosen position. The goal was to identify the best position of the sensor to capture the information and to minimize the number of sensors as much as possible.

To avoid the perturbation resulting from the reflected waves, an algorithm in MATLAB software was developed to select the peaks of only the first received signals, as can be shown in Figure 2. The locations of the chosen sensors are detailed in Table 3.

Table 3. The positions of the sensors from the transverse defect and the excitation spot zone.

\begin{tabular}{lcccc}
\hline & Sensor $\mathbf{1}$ & Sensor $\mathbf{2}$ & Sensor $\mathbf{3}$ & Sensor 4 \\
\hline Distance from the transverse defect & $15 \mathrm{~mm}$ & $30 \mathrm{~mm}$ & $60 \mathrm{~mm}$ & $90 \mathrm{~mm}$ \\
Distance from the excitation zone & $50 \mathrm{~mm}$ & $65 \mathrm{~mm}$ & $95 \mathrm{~mm}$ & $125 \mathrm{~mm}$ \\
\hline
\end{tabular}
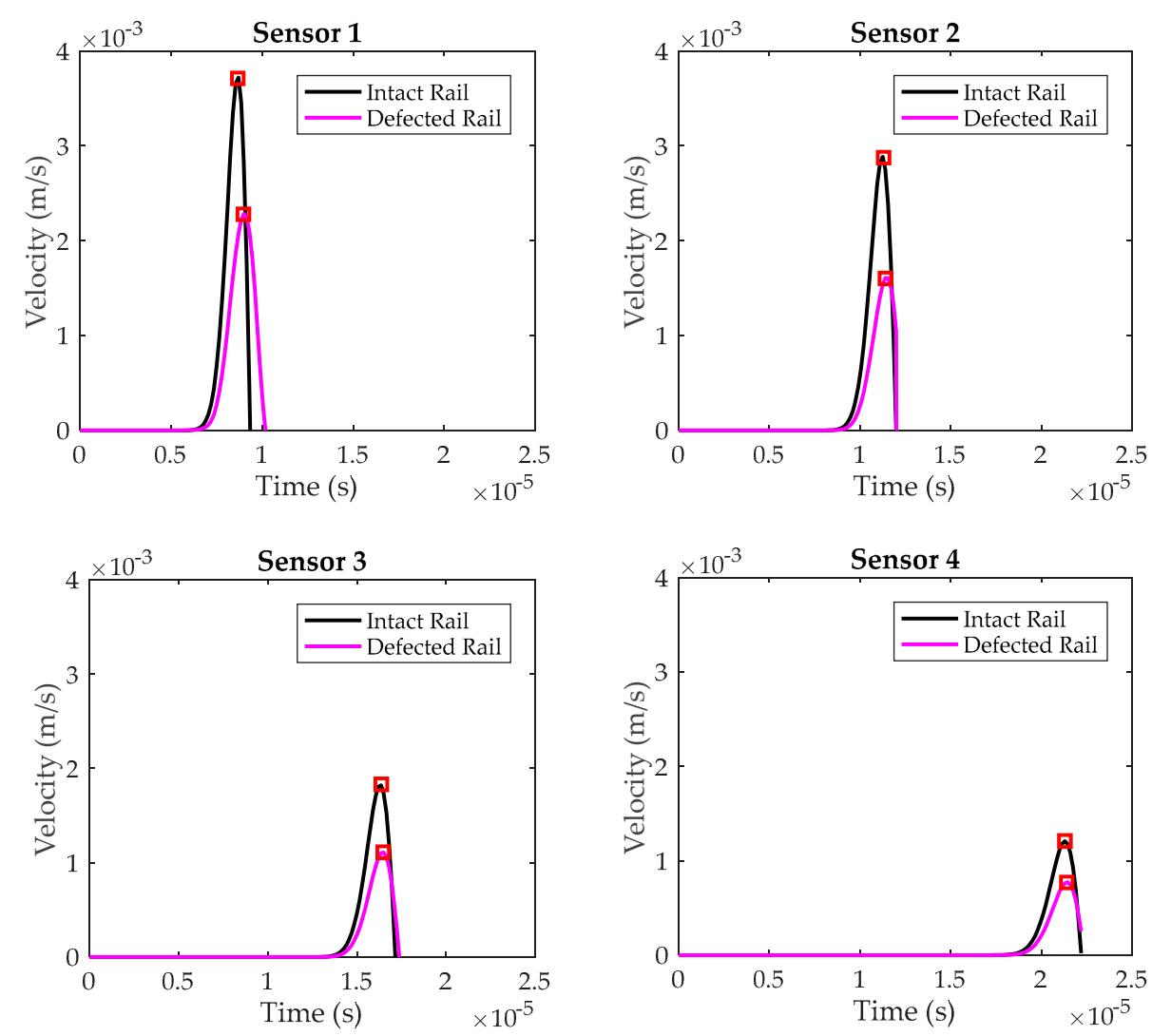

Figure 2. The results of the four sensors, for the case of rail with transverse defect and intact rail. 
The velocity of the propagated wave decreased in the presence of the defect, as can be shown in Figure 2. Sensor 1 can capture the signal faster than the other sensors, because of the distance that separates the position of the sensors from the defect and the spot excitation area, as can be seen in Table 3. The signal amplitude increased when the sensor position was closer to the defect. These results indicate that the location of the sensors has a significant effect on the amplitude. As a result, the number of sensors can be reduced since sensors 3 and 4 capture the signal later than the first two sensors; and the amplitude for both the intact and defected rail decreases.

\section{Conclusions}

The most important fact revealed in this study is the influence of the position of the sensors on the signal amplitude. Each sensor captures information depending on the distance that separates the sensor from the defect and the spot area, and once the distance changes, the influence of the amplitude also changes. The obtained results have enabled us to determine the best arrangement of sensor positions to operate the detection of transverse rail defects.

\section{References}

1. Scalea, F.; Rizzo, P.; Coccia, S.; Bartoli, I.; Fateh, M.; Viola, E.; Pascale, G. Non-contact ultrasonic inspection of rails and signal processing for automatic defect detection and classification. Insight-NDT and condition monitoring. Insight Non-Destr. Test. Condition Monit. 2005, 45, 346-353.

2. Toliyat, H.A.; Abbaszadeh, K.; Rahimian, M.M.; Olson, L.E. Rail defect diagnosis using wavelet packet decomposition. IEEE Trans. Ind. Appl. 2003, 39, 1454-1461.

3. Cerniglia, D.; Garcia, G.; Kalay, S. Application of laser induced ultrasound for rail inspection. In Proceedings of the Seventh World Congress on Railway Research (WCRR), Montreal, QC, Canada, 4-8 June 2006.

4. Rizzo, P. Sensing solutions for assessing and monitoring railroad tracks. In Sensor Technologies for Civil Infrastructures; Woodhead Publishing Limited: Cambridge, UK, 2014.

(C) 2019 by the authors. Licensee MDPI, Basel, Switzerland. This article is an open access article distributed under the terms and conditions of the Creative Commons Attribution (CC BY) license (http://creativecommons.org/licenses/by/4.0/). 